\title{
Gradhiva
}

GRADHIV

Revue d'anthropologie et d'histoire des arts

26 | 2017

En croire ses sens

\section{La main sur l'écran. Pentecôtisme et expérience du « toucher » télévisuel}

A Hand on the Screen. Pentecostalism and the Experience of Television "Touch"

Damien Mottier

\section{(2) OpenEdition}

1 Journals

Édition électronique

URL : http://journals.openedition.org/gradhiva/3420

DOI : $10.4000 /$ gradhiva.3420

ISSN : 1760-849X

Éditeur

Musée du quai Branly Jacques Chirac

Édition imprimée

Date de publication : 6 décembre 2017

Pagination : 22-45

ISBN : 978-2-35744-096-8

ISSN : 0764-8928

Référence électronique

Damien Mottier, «La main sur l'écran. Pentecôtisme et expérience du « toucher » télévisuel », Gradhiva [En ligne], 26 | 2017, mis en ligne le 06 décembre 2019, consulté le 03 janvier 2020. URL : http:// journals.openedition.org/gradhiva/3420; DOI : 10.4000/gradhiva.3420

(c) musée du quai Branly 


\section{La main sur l'écran}

Pentecôtisme et expérience du «toucher» télévisuel

par Damien Mottier

Apparu aux États-Unis dans les années 1950 avec les premiers sermons télévisés, le geste de poser la main sur l'écran d'un téléviseur afin de recevoir l'effusion de l'Esprit saint qu'un télé-prédicateur promet d'activer est caractéristique de l'expérience pentecôtiste. Selon cette grammaire communicationnelle, il s'agit de transformer la personne par incorporation de l'Esprit saint, sinon de provoquer sa conversion ou sa guérison. Ce geste, qui fait de l'engagement des corps dans un régime d'images télévisuelles un acte fort de conversion, n'est pourtant pas en soi innovant. II s'agit au contraire d'un geste anachronique de la modernité pentecôtiste qui actualise et réinvente toute une série de pratiques religieuses de contact avec les images. Conçu comme une variation sur les processus historiques de formation télévisuelle de l'expérience pentecôtiste, cet article vise à penser la main posée sur l'écran comme un motif de l'Incarnation et une reformulation de la logique miraculeuse du touché-touchant qui est au principe du christianisme. 
1. Rappelons que le pentecôtisme est un mouvement protestant évangélique. En référence à la Pentecôte, il se définit par le baptême de l'Esprit saint, qui confère à ceux qui l'expérimentent différents dons (parler en langues, etc.), et se caractérise par la combinaison de trois éléments fondamentaux: l'expérience émotionnelle de la présence divine et de son efficacité, la référence privilégiée à la Bible et le caractère professant du groupement religieux (Willaime 1999: 9).

2. Thomas Csordas par exemple, s'est intéressé aux « images sensorielles " et aux "modes d'attention somatique" associés aux rituels de guérison du renouveau charismatique $(1997,2002)$ tandis que Tanya Lurhmann, interrogeant les modalités perceptives de l'expérience charismatique à partir du mouvement évangélique nord-américain Vineyard, a récemment forgé I'hypothèse phychologique de «l'absorption» (2012).

Sur l'analogie avec le couran électrique au sein des pentecôtimes, voir notamment De Abreu 2009. Et sur cette expérience du toucher: De Witte 2011a, 2011b.
3. En témoigne le scandale provoqué en 2007 par un prédicateur ghanéen qui a importé des États-Unis une "machine à toucher électrique" (electric touch machine) pour simuler l'action du Saint-Esprit et leurrer ses fidèles (De Witte 2013).

4. Sur cette « haptique générale" du touchétouchant, je renvoie à la relecture du travail de Jean-Luc Nancy par Jacques Derrida (2000). Quant aux rapports entre anthropologie des images et anthropologie et théologie chrétiennes, "fondés sur une conception de l'homme créé ad imagem De et promis au salut par l'Incarnation du Christ imago Patris " (Schmitt 2002: 54) je m'appuierai sur les travaux de Belting 2004, Didi-Huberman 2007 et Schmitt 2002.
Cet article est consacré à un geste banal de l'expérience pentecôtiste: poser la main sur l'écran d'un téléviseur ou d'un ordinateur afin de recevoir l'effusion du Saint-Esprit qu'un téléprédicateur promet d'activer. Une telle pratique de contact peut surprendre. Le toucher digital est certes devenu chose banale ces dernières années avec la multiplication des tablettes et des smartphones, mais l'application ou l'orientation de la paume de la main vers un écran relève d'un usage peu conventionnel de la médiation télévisuelle. Selon la virtuosité de cette grammaire communicationnelle, il s'agit de transformer la personne par incorporation de l'Esprit saint ${ }^{\mathbf{1}}$ que libère la performance du téléprédicateur, sinon de provoquer sa guérison ou sa conversion.

Apparue aux États-Unis dans les années 1950 avec les premiers sermons télévisés, cette implication des corps dans un régime d'images animées et sonores est une épreuve décisive de l'expérience pentecôtiste. La plupart des fidèles se disent touchés, non pas métaphoriquement mais physiquement, comme traversés par une déflagration intérieure. L'analogie avec le courant électrique est fréquente et plusieurs chercheurs ont fondé leur étude des phénomènes de guérison miraculeuse ou de communication avec le divin en s'attachant à l'analyse des registres sensoriels ${ }^{2}$. En complément de l'intérêt porté aux discours des acteurs, la compréhension de cette expérience du toucher télévisuel est nécessairement à inscrire dans une ethnographie de l'action cérémonielle, à laquelle participe l'économie de la télévision. Et c'est à travers l'analyse combinée du complexe de relations engagées de part et d'autre de l'écran, des caractéristiques du médium, des catégories sociales de perception et des modalités d'activation de l'agir divin que cette singulière articulation entre fait d'image (télévision) et fait religieux (pentecôtiste) doit être saisie.

Tous les individus ne sont pourtant pas affectés par cette pratique de contact télévisuel. Soupçonneux vis-à-vis du rôle d'activateur de charisme des téléprédicateurs, certains pentecôtistes la dénigrent même comme une conduite inappropriée. Sans insister sur la crainte de la simulation et du simulacre, jamais totalement dissipée ${ }^{3}$, je débuterai par une situation de mise en échec de cette performance télévisuelle. L'intérêt de cette situation, qui ne relève pas d'une observation directe mais d'un souvenir d'enfance qu'un proche m'a rapporté, est double: faire de la conversion des corps le principal enjeu de ce dispositif d'effraction du téléprédicateur au domicile des fidèles et nous projeter dans l'Amérique des années 1950, au moment précis où ce jeu de main est devenu caractéristique des pentecôtistes. Ainsi, déroutant les lignes de l'espace et du temps - de l'Amérique des années 1950 à l'essor médiatique des pentecôtistes africains contemporains en passant par une ville-monde des années 1920, Los Angeles, où de nouveaux modes de prédication ont été élaborés en relation avec l'industrie du film naissante cet article se présente comme une variation sur les processus de formation télévisuelle de l'expérience pentecôtiste. II est fondé sur l'idée que le travail des médiations techniques est constitutif de l'expérience religieuse et vise à penser ce geste comme un motif de l'Incarnation et une reformulation de la logique miraculeuse du touché-touchant ${ }^{4}$ qui est au principe du christianisme. 


\section{Un geste d'ouverture}

Le 2 juin 1961, comme tous les premiers vendredis du mois, Richard $\mathbf{5}$, âgé de 11 ans, regardait en famille l'une des émissions emblématiques de la Radiodiffusion-télévision française (RTF): “Cinq colonnes à la une ». Parmi les reportages diffusés en noir et blanc, l'un d'eux a particulièrement frappé son esprit. Intitulé «Le chapiteau des miracles», ce reportage de dix minutes présentait pour la première fois en France l'activité d'un célèbre prédicateur américain, Oral Roberts, qui sillonnait les États-Unis et organisait sous chapiteau des campagnes d'évangélisation insistant sur la promesse de guérison miraculeuse ${ }^{6}$. Fondateur en 1947 de l'Oral Roberts Evangelistic Association, Roberts est l'un des «prédicateurs guérisseurs " (faith healers) qui ont contribué à l'élaboration et à la propagation du modèle télévangéliste. II s'appliqua dès 1954 à la transmission de ses sermons dans le but, disait-il, d'installer les téléspectateurs au «premier rang des miracles » (Harrell 1985: 125-130). Produit directement par ses équipes dans le cadre de «The Abundant Life Program », une émission hebdomadaire retransmise à partir de 1958 sur un réseau de chaînes couvrant l'essentiel du territoire américain, le document diffusé par «Cinq colonnes à la une » s'accompagnait du commentaire d'un journaliste français qui ne cherchait pas à dissimuler son étonnement à l'égard de cette pratique pentecôtiste mal connue en France ${ }^{7}$ :

Non ce n'est pas de la fiction, c'est la réalité. Si vous étiez Américain, vous verriez cette émission après-demain dimanche sur votre écran de télévision. Chaque dimanche, sous le chapiteau d'un cirque, en direct d'une ville des États-Unis, Oral Roberts lit la Bible devant dix mille personnes. Sa firme a son sigle: Oral Roberts Evangelistic Association. Et son siège social est à Tulsa dans l'Oklahoma. C'est un important building où de jour et de nuit, sur un simple coup de téléphone de votre part, des prieurs de garde peuvent prier pour vous. Ses publications tirées à des millions d'exemplaires et pour des lecteurs de tous les âges racontent les paraboles de l'Évangile en bande dessinée. Après tout, pourquoi n'y aurait-il pas des comics sacrés, comme il y a de la musique sacrée? La doctrine d'Oral Roberts, que Dieu a bien voulu lui révéler personnellement certaines nuits de guérison, est simple: elle tient tout entière dans le pouvoir de ses mains, capables, comme celles du Christ dont il n'est en quelque sorte que le démarcheur, de guérir et d'imposer la foi. Les mains, toutes les mains, jouent d'ailleurs un grand rôle dans les réunions d'Oral Roberts, et ses fidèles sont souvent invités à les joindre ou à les lier pour établir ce qu'il appelle les «points de contact»: «Par les points de contact, vous pouvez vous frayer un chemin vers Dieu. Ainsi vous pourrez recevoir Dieu dans votre âme et dans votre corps. II sera plus proche de vous que le souffle dans vos narines. ”

Le reportage s'attarde sur différentes situations d'imposition des mains qui concluent le sermon de Roberts. Assis sur une chaise pliante au bord de l'estrade, en pantalon sombre, chemise blanche et cravate, Roberts reçoit individuellement (parfois en couple) les candidats au miracle qui se pressent en file indienne au-devant de lui. Leur tête est à hauteur de son buste. II leur demande de se présenter, récapitule et expose publiquement, à l'aide des fiches que ses assistants ont rédigées, les maux dont ils souffrent - problème
5. C'est ainsi que je nommerai celui qui m'a rapporté cette expérience.

6. Ce reportage est consultable en libre accès sur le site de l'Institut national de l'audiovisuel (INA) : http://www.ina.fr/ video/CAF93014119.

7. Pour donner un élément de comparaison, la première messe a été télévisée en France à Noël 1948. Pour une analyse contextualisée des enjeux catholiques de la christianisation des usages de la télévision, voir Leventopolous 2011. 
8. Je reprends ici, ainsi que dans la suite du texte, la traduction du commentateur en voix off.

9. Pour une analyse contextualisée du toucher dans la culture américaine, voir Chidester 2000 et 2005

\section{ci-contre}

fig. $1 \mathrm{a}$ et $1 \mathrm{~b}$

Oral Roberts,

photogrammes extraits

du reportage "Le chapiteau

des miracles", Cina

colonnes à la une.

ORTF, 2 juin 1961. DR. de dos, surdité, bégaiement, arthrose, etc. Puis il commence à prier pour chacun d'eux avec autorité: "Mon Dieu nous prions pour Norman W. du Tennessee. Pour que la santé lui soit rendue. Pour qu'il soit guéri par la main qui a guéri les gens il y a deux mille ans. Que son dos et ses jambes soient libérés et guéris ${ }^{8}$. " Les gestes de ses mains alternent les positions en rythme avec les modulations de sa voix. Roberts enveloppe le visage de cet homme sans le toucher, puis il applique brusquement sa main droite sur le front et revient à la position initiale. La performativité de la Parole de Dieu qu'il convoque par une forte prière est ainsi traduite par ce jeu de mains alternant douceur et autorité.

Au terme de chaque manipulation, Roberts sollicite les fidèles pour témoigner instantanément du miracle de la guérison qui vient de s'accomplir: "Alors, monsieur W., comment savez-vous que Dieu a déjà commencé à vous guérir? - La douleur est partie de mes épaules. - Et votre dos? - Plus de douleur dans le dos. - Et le reste? - Plus de douleur dans le poignet. - Retournez voir votre docteur, monsieur W., vous êtes guéri. " Les fidèles se succèdent. «Madame, avez-vous senti le Seigneur? Oui elle l'a senti partout. Votre impression? - Formidable. " Tout au long de sa carrière, Roberts a appliqué sa main sur le front de plusieurs centaines de milliers d'individus (deux millions selon ses biographes). La retransmission télévisuelle de cette pratique de $\operatorname{contact}^{9}$ a significativement contribué à la propagation de l'imaginaire miraculeux de l'agir divin. Mais surtout, cette technique corporelle d'imposition des mains a rapidement été soumise à l'épreuve de l'écran, devenu comme par transparence le cadre d'une expérience médiatique du toucher miraculeux. En épilogue de ce reportage, les télé-spectateurs sont ainsi invités à appliquer directement leur main sur celle de Roberts, dans une forme de corps-à-corps avec lui (fig. 2b):

C'est le final, l'apothéose. Le révérend Roberts va poser sa main miraculeuse sur une plaque de verre. Ce qu'elle a fait pour les fidèles qu'elle a touchés réellement, pourquoi ne le pourrait-elle pour les millions de télé-spectateurs qui ont besoin de son secours? Alors en cette minute, partout dans les États-Unis, des milliers d'hommes et de femmes se lèvent et vont imposer leurs mains bien à plat contre la vitre froide de leur écran de télévision. Jusqu'à toucher doigt contre doigt, paume contre paume, cette main miraculeuse. Après tout, si le cœur vous en dit, essayez!

Richard, qui regardait ce reportage en famille, a donc essayé en ce vendredi 2 juin 1961, appliquant consciencieusement sa main sur l'écran bombé d'un téléviseur noir et blanc qui se trouvait dans son salon. II souffrait d'asthme chronique et espérait en guérir. Son père, non pratiquant, catholique par tradition plus que par conviction, lui avait recommandé de faire ce geste, "sait-on jamais». Si l'écran ne s'est pas «ouvert» pour Richard sur le miracle promis, cette mise en échec de la relation charismatique ne peut être réduite au simple constat du «ça marche " ou «ça ne marche pas", ni du manque de conviction avec lequel il a peut-être exécuté ce geste. 

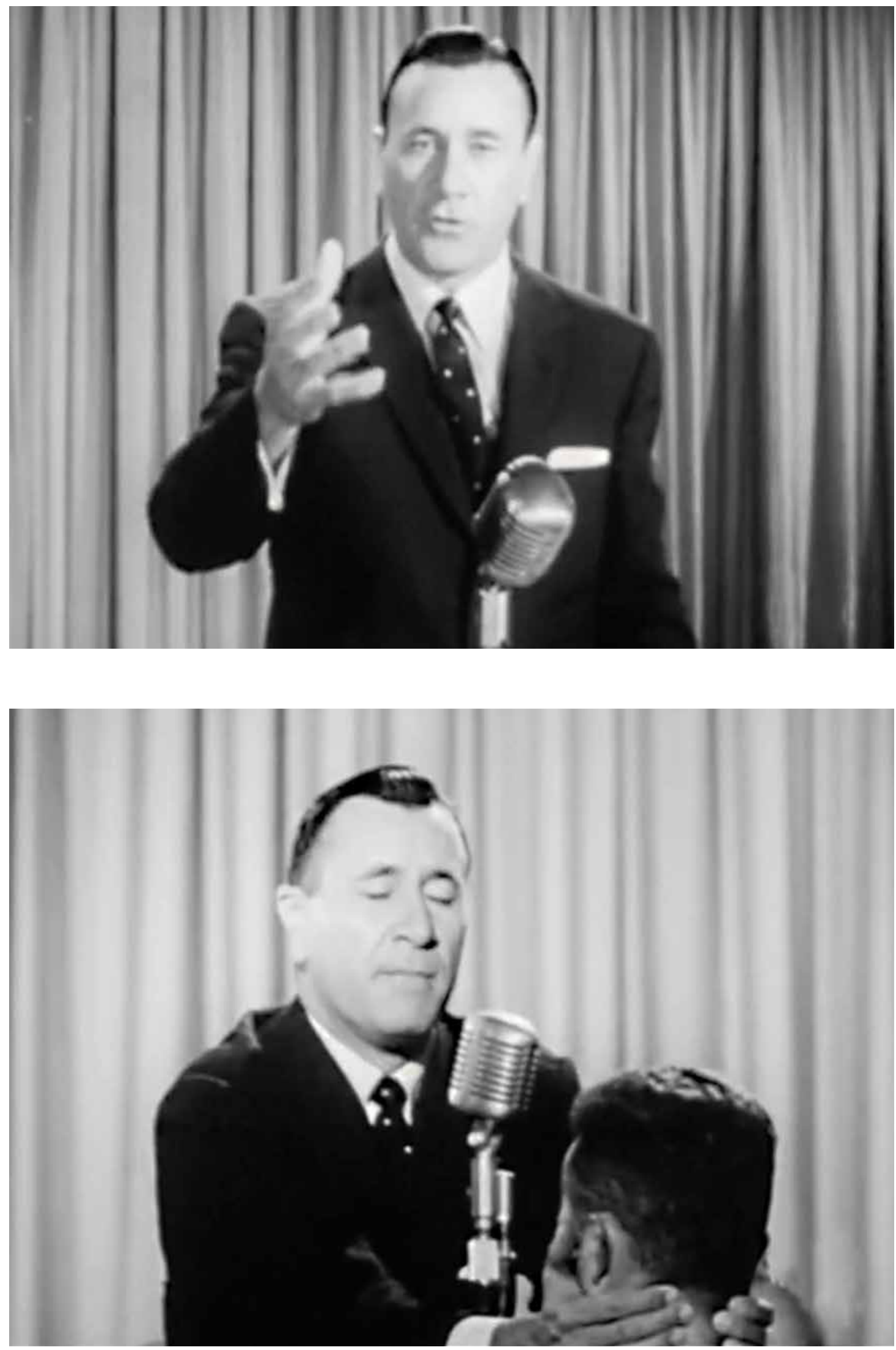

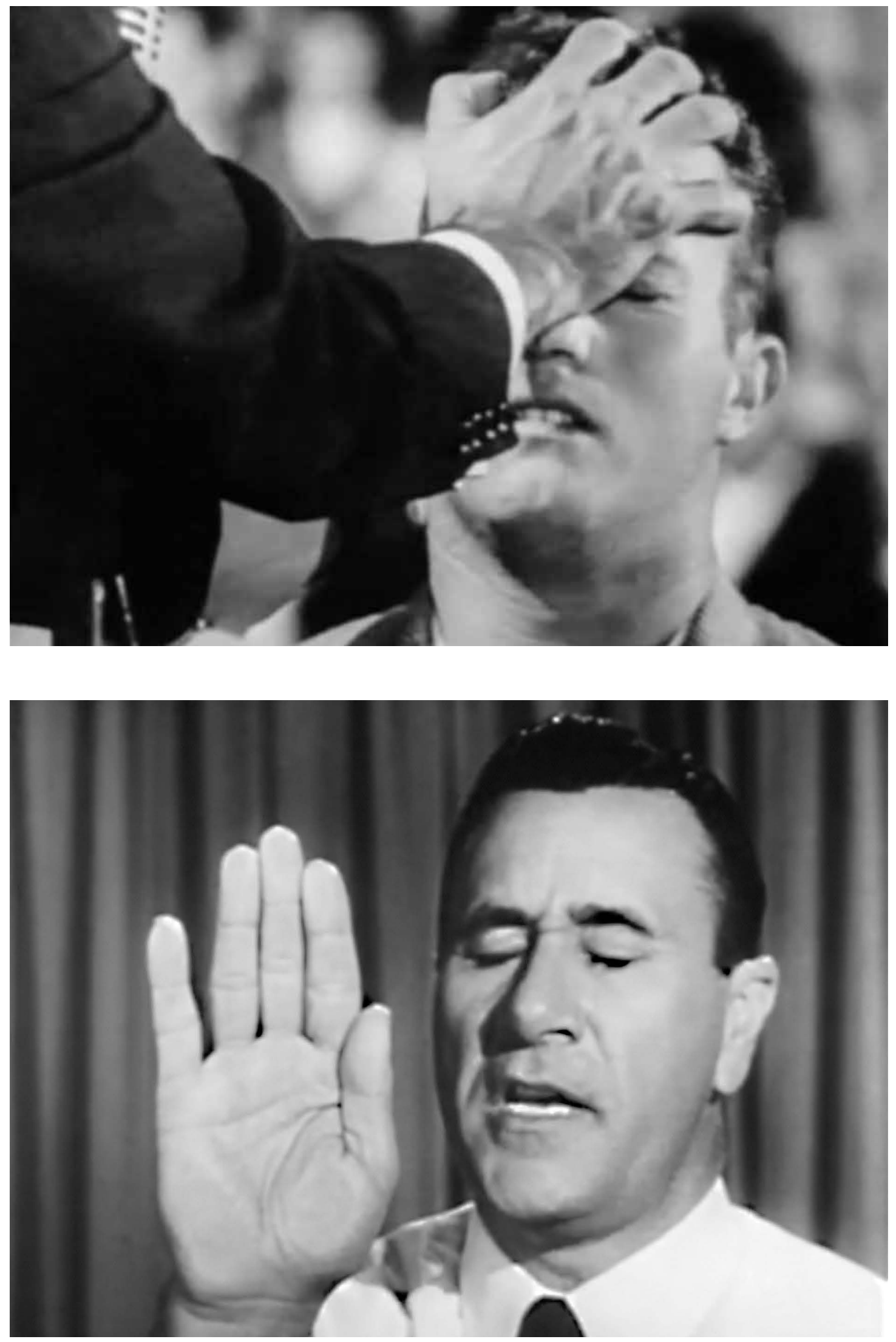
Suivant l'hypothèse du «déclencheur social » (social kindling) avancée par Tanya Luhrmann, une socialisation préalable au sein de cet univers pentecôtiste, dont Richard ignorait tout ou presque et qu'il a regardé avec une certaine incompréhension, l'aurait sans doute aidé à mieux appréhender cette pratique de contact. À moins que cette exposition brutale ne provoque en lui une sensation inédite qui le conduise ultérieurement à socialiser cette expérience au sein de cet univers religieux, comme en témoignent certains cas de conversions télévisuelles. Qu'elle soit première ou seconde, la socialisation est nécessaire pour habituer le corps à répondre à ce type de sollicitation - «convertir les sens », écrit Marleen de Witte (2011a) - et à s'engager dans la répétition de cette expérience du toucher télévisuel: «L'expérience résulte toujours d'une interaction entre attente individuelle, sollicitation culturelle, pratique religieuse et sensibilité corporelle " (Cassaniti et Luhrmann 2014: 341 , traduction de l'auteur). Mais ce qui m'intéresse particulièrement ici est la dialectique d'ouverture et de fermeture dont Georges Didi-Huberman a fait une caractéristique majeure de notre rapport aux images. «Nous sommes devant les images, écrit-il, comme devant d'étranges choses qui s'ouvrent et se ferment alternativement à nos sens - que l'on entende dans ce dernier mot un fait de sensation ou un fait de signification, le résultat d'un acte sensible ou d'une faculté intelligible.» Cette dialectique est autre chose qu'une simple «métaphore» ou un «pure principe de réception»: un «fait de structure», un «événement d'image », un «principe d'animation », «un processus d'altération» (Didi-Huberman 2007: 25-33). La main posée sur l'écran est un geste d'ouverture. Mais l'image animée de Roberts et de sa main droite miraculeusement tendue vers les téléspectateurs a échappé à l'appréhension de Richard qui s'est retiré de l'écran sans se sentir «touché ", c'est-à-dire modifié, transformé par cette étreinte télévisuelle. II s'est soumis au dispositif sans parvenir à «ouvrir» l'image.

\section{Culture du spectacle}

Il serait difficile de comprendre les sens de ce geste d'ouverture sans l'inscrire dans une histoire plus longue des modalités de mise en scène de l'expérience charismatique. L'art de la prédication - oralité, gestualité, etc. - est un exercice de la scène. C'est sur scène - en plein air, sous chapiteau, dans un espace de culte ou une salle louée et aménagée pour la circonstance que le pouvoir des prédicateurs se manifeste et qu'il est soumis à l'épreuve des fidèles. Les télévangélistes, dont Roberts est une figure majeure, ont ainsi hérité du dialogue noué de longue date entre performance charismatique et arts du spectacle. La prédicatrice Aimée Semple McPherson par exemple, à laquelle l'historien Matthew Sutton a consacré une remarquable biographie (2007), a marqué le développement du pentecôtisme en réalisant, dès les années 1920, une étonnante synthèse entre prédication et divertissement. Un retour sur les innovations de cette femme, dont le sens de la mise en scène en a fait l'une des personnalités les plus célèbres des États-Unis de l'entre-deux-guerres, permet de prendre la mesure de ces affinités.

Née en 1890 au Canada, McPherson a créé à Los Angeles l'International Church of the Foursquare Gospel, après plusieurs années d'une prédication itinérante à travers le continent nord-américain qui l'a conduite à improviser ici et là, dans la tradition revivaliste, des prédications en plein air ci-contre

fig. 2 a et $2 b$

Oral Roberts,

photogrammes extraits

du reportage "Le chapiteau

des miracles", Cinq

colonnes à la une,

ORTF, 2 juin 1961. DR. 
10. Cette formule est un condensé de la doctrine pentecôtiste (Willaime 1999: 7).

\section{ci-contre}

fig. 3

International Newsreel Corp., New York.

Aimée Semple McPherson combat «Godless Gorilla », 23 janvier 1934. Avec l'aimable autorisation de Oregon Historical Society. ou sous chapiteau. L'église "quadrangulaire" Foursquare repose sur ce qu'elle présente comme les quatre piliers de la foi: Jésus "sauve», "guérit», «baptise» et «revient bientôt ${ }^{10}$ ». Un temple de cinq mille trois cents places, l'Angelus Temple, dont l'architecture ressemble à celle d'un amphithéâtre avec son orchestre, ses balcons, ses coulisses et sa scène majestueuse, a été construit à sa demande et inauguré le $1^{\text {er }}$ janvier 1923. Rapidement, Mcpherson y a enchaîné les sermons. Elle prêchait en alliant les mises en scène de théâtre ou de music-hall à des lectures littéralistes de la Bible. La proximité de l'Angelus Temple avec l'industrie naissante du film à Hollywood (fig. 3) a facilité la mise en œuvre concrète de cette nouvelle manière de concevoir la prédication: «Par son positionnement dans la capitale mondiale du show business, McPherson a changé la manière dont la religion américaine était pratiquée. " (Sutton 2007: 4, traduction de l'auteur) Elle a notamment mobilisé les compétences professionnelles de certains de ses fidèles travaillant à Hollywood comme décorateurs, maquilleurs ou comédiens afin de concevoir et d'interpréter ses fameux sermons illustrés (illustrated sermons), qui mettaient en scène chaque dimanche des épisodes de l'Évangile ou de sa propre vie. D'après Sutton, ses sermons étaient le «meilleur spectacle» de Los Angeles, des shows qui n'avaient rien à envier à ceux de Broadway. Au cours de l'un d'eux, intitulé "Arrêtée pour excès de vitesse" (Arrested for speeding), Mcpherson est arrivée à l'Angelus Temple en moto, vêtue d'un uniforme de policier, une casquette sur le crâne, en lançant à la foule: «Vous êtes sur la route de l'enfer ". L'objectif de ces spectacles était selon ses propres dires d'illustrer la Parole de Dieu, de la mettre en action et non plus seulement de la prêcher, «de manière à ce que les yeux puissent voir aussi bien que les oreilles entendent» (ibid.: 71, traduction de l'auteur).

Intrigués par le style de cette femme, qui soignait son apparence en portant des tenues vestimentaires (hermine, fourrure, etc.) dignes des stars hollywoodiennes, plusieurs personnalités de l'industrie du film sont régulièrement venues à l'Angelus Temple. Charlie Chaplin assistait occasionnellement à ses prestations, prodiguant quelques conseils de mise en scène à cette prédicatrice qu'il considérait comme une «actrice» (ibid.: 75-76). L'audace de Mcpherson, sa beauté et, plus tard, le scandale de sa disparition avec son amant, n'ont pas tardé à être adaptés au cinéma. En 1931, Franck Capra s'est inspiré de son histoire dans The Miracle Woman. Cette fiction, dans laquelle Barbara Stanwyck prêche dans une cage entourée de fauves, a été suivie de plusieurs autres. Les facéties de Mcpherson et les controverses qui ont émaillé sa vie personnelle ne doivent toutefois pas faire oublier l'essentiel. Sans jamais dévier d'une lecture fondamentaliste des Évangiles, elle a su faire des arts de la scène et des nouveaux moyens de communication - elle fut la première femme à prêcher sur les ondes radiophoniques - un élément constitutif de sa performance. «McPherson est la première célébrité religieuse de l'ère des mass media. Couplée à un credo conservateur, son appropriation des derniers outils médiatiques a créé un précédent pour les prédicateurs les plus populaires du xx siècle, de Billy Graham et Oral Roberts à Pat Robertson. " (ibid.: 3-4, traduction de l'auteur) À quelques encablures d'Hollywood, la scène de l'Angelus Temple est ainsi devenue le laboratoire d'une nouvelle manière de concevoir l'exercice de la prédication, créant les conditions durables d'une connivence entre industrie du spectacle et expérience pentecôtiste qui n'a cessé, depuis lors, de se développer. 


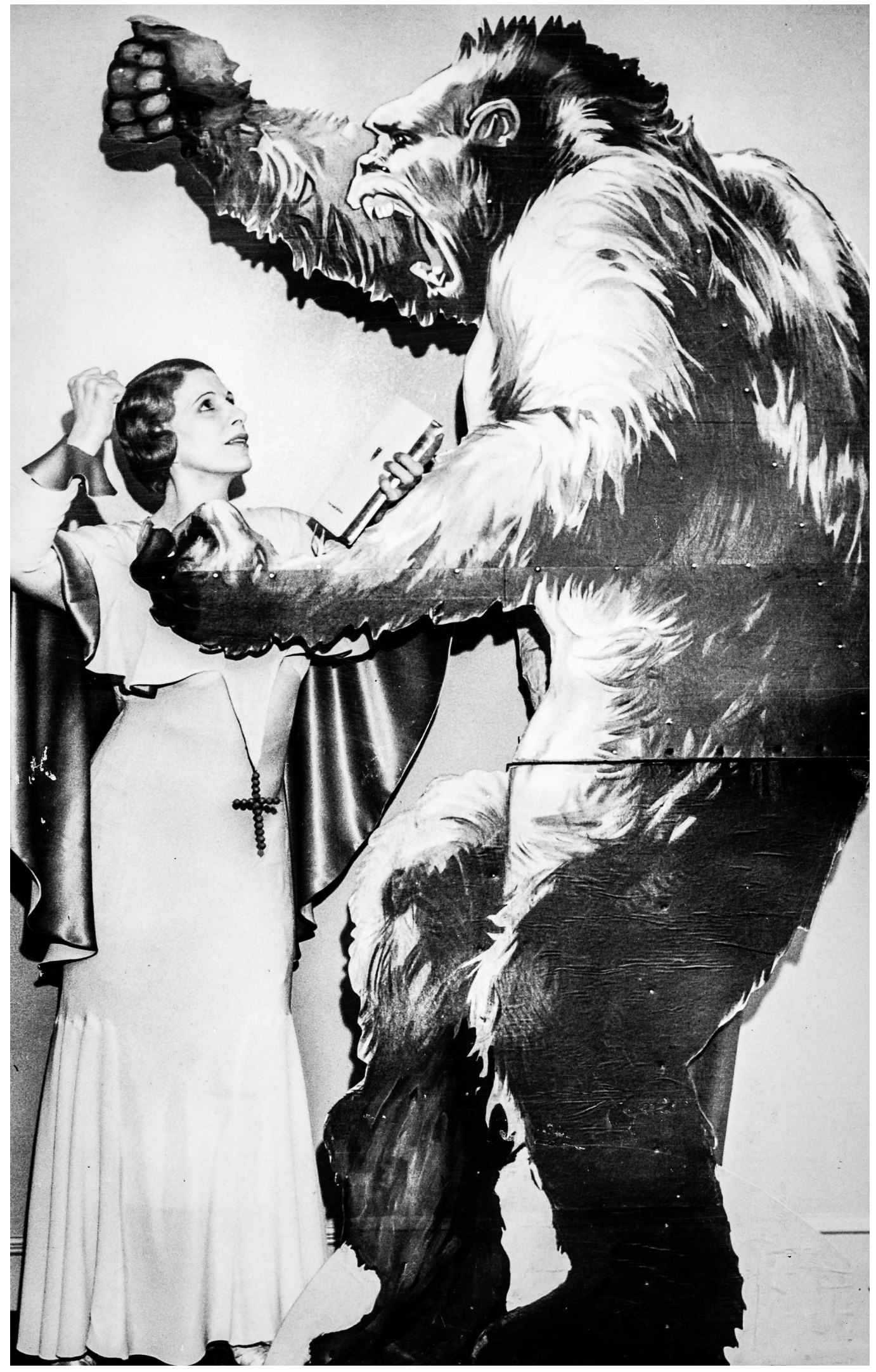




\section{LA ROSE POURPRE DU CAIRE}

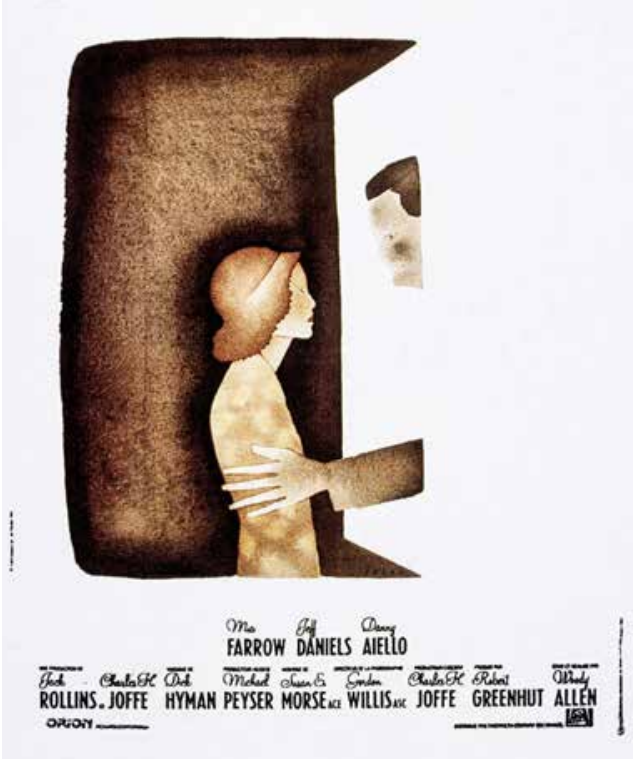

fig. 4

Jean-Michel Folon Affiche pour La Rose pourpre du Caire, réal. Woody Allen, 1985. Photo Rue des Archives (C) Folon/ADAGP, Paris, 2017.

\section{Formation télévisuelle}

«Pionnier de la guérison miraculeuse à la télévision 》 (Harrel 1985 : 127-128, traduction de l'auteur), Roberts s'est inscrit dans la continuité des innovations scéniques d'Aimée Semple McPherson. Ses premières prédications télévisées ont été enregistrées en studio, dans des conditions proches des sermons radiophoniques, sans que cela lui donne satisfaction. Le fait que les contraintes du studio le séparent physiquement de la scène - «tentes-cathédrales » ou « chapiteaux» - sur laquelle il avait l'habitude de se produire entravait d'après lui l'expression de sa performance. Au prix d'une prouesse technique remarquable pour l'époque, il a donc pris la décision de filmer ses sermons directement au cours de ses campagnes d'évangélisation: "Trois films pilotes ont été réalisés à Akron pour un montant de 42000 dollars. Le résultat fut si impressionnant que Roberts a été amené à reconsidérer l'ensemble de sa conception des usages de la télévision. Non seulement les sermons avaient été filmés, mais aussi les appels à la repentance, les files de fidèles en attente de guérison, les nombreux miracles, les allées et venues d'une foule immense, la réaction de l'assemblée - tout cela était présent dans les films. » (ibid.: 127, traduction de l'auteur) L'immixtion des tournages au sein de l'espace cultuel produisit donc l'effet escompté en redonnant à Roberts le sens de la relation avec les fidèles. Restait cependant à convaincre les sceptiques du bien-fondé d'une telle médiatisation qui visait à rendre ses sermons accessibles au grand public et à assurer sa propre promotion, mais aussi à toucher les téléspectateurs et à transformer leur manière de concevoir les modalités d'action de l'Esprit saint.

Roberts a pris soin, dans un premier temps, de faire authentifier ses prestations télévisuelles. Harell relate ainsi que certains «telecast » étaient précédés par la présentation d'un «document notarié » signé de la main d'un «juge local». Présent lors du tournage, ce dernier «jurait que "les événements filmés avaient réellement eu lieu sur place au cours de réunions organisées sous une immense tente cathédrale" » (ibid. : 127, traduction de l'auteur). Les témoignages des téléspectateurs ont ensuite rapidement joué leur rôle; le journal local de Wichita Falls, au Texas, a par exemple consacré sa une du 2 mai 1955 au cas d'une femme miraculeusement guérie de paralysie par la prière télévisée de Roberts (ibid.: 128). Enfin, l'efficacité de cette transmission télévisuelle a été formalisée par ce geste d'ouverture qui perfore l'écran et fait tomber, à sa manière, le «quatrième mur ${ }^{\mathbf{1 1}}$ ». En orientant la paume de sa main vers l'objectif de la caméra, Roberts offrait aux télé-spectateurs un «point de contact» à travers lequel le Saint-Esprit pouvait se manifester miraculeusement en eux. Ce jeu de mains marque un changement de sensibilité important. II n'est plus seulement question de prêcher à la télévision, de convaincre ou d'appeler à la repentance, mais de transmettre directement le message en provoquant la manifestation de l'Esprit saint dans le corps des fidèles par la vertu du téléprédicateur. Plus qu'un symbole, cette «formule d'émotion ${ }^{12}$ » est devenue un symptôme de l'expérience télévisuelle des pentecôtistes.

Si ce geste d'ouverture, repris d'innombrables fois, s'est imposé comme un acte fort de conversion au mode d'expérimentation pentecôtiste de l'agir divin, le modèle télévangélique a également permis d'accroître, plus simplement, la visibilité de cette pratique religieuse. Les sermons de Roberts ont rapidement été télévisés hors du territoire américain, au Canada et aux 
Philippines dès 1957, puis en Afrique: au Ghana, par exemple, à la fin des années 1970 tandis que le Nigérian Benson Idahosa, premier télévangéliste africain formé par Roberts, lançait son programme «Redemption Hour » sur le modèle de «The abundant Life Program »13. Combinée à la dynamique des missions et des croisades dont les prédicateurs américains se sont servis pour atteindre tous les continents, la médiation télévisuelle a contribué à l'accélération des processus de globalisation. La retransmission des sermons, la commercialisation des cassettes vidéographiques et la progressive libéralisation des médias ont même joué un rôle d'autant plus important dans la création des Églises pentecôtistes locales, africaines notamment, que ces visions techniquement animées ont été employées comme «matériel pédagogique " par des prédicateurs locaux. Plusieurs fondateurs d'églises pentecôtistes à Kinshasa durant les années 1980 et 1990 m'ont ainsi rapporté qu'ils avaient concrètement assuré une partie de leur formation pratique - learning to do rather than learning to think (« apprendre à faire plutôt qu'apprendre à penser») (Lurhmann 2012: xxI) - en visionnant des vidéos de sermons. Les différents supports de la médiation télévisuelle ont donc favorisé la transportabilité (Csordas 2009) de cette pratique religieuse et accompagné, par imprégnation, l'éclosion de toute une génération de fondateurs d'Églises, contribuant à la formation de leur sensibilité pentecôtiste.

Plus récemment, en région parisienne au milieu des années 2000, j'ai rencontré plusieurs jeunes prédicateurs qui, tout en étant formés dans les églises et en fréquentant des lieux de culte, faisaient leur apprentissage par le truchement de vidéos de prédicateurs auxquels ils s'identifiaient. À force de visionner ces vidéos, certains finissent par incorporer le style des prédicateurs avec lesquels ils n'entretiennent pourtant, le plus souvent, qu'une relation en miroir. Cette initiation médiatique à l'expression de leur charisme procède par appropriation sélective de gestes, de postures, d'intonations, de la rythmique et de la rhétorique, si bien que les continuités de style qui imprègnent leurs corps contribuent, dans certaines circonstances, à la production de régimes de filiation spirituelle identifiés par les fidèles ${ }^{\mathbf{1 4}}$. Dès lors que l'animation télévisuelle est susceptible d'alimenter une telle idiosyncrasie du comportement, on peut se demander si elle ne constitue pas, à travers l'infini miroitement des écrans, une forme de liturgie sur laquelle l'orthopraxie de cet univers religieux, par ailleurs extrêmement fragmenté, est en partie fondée.

Enfin, les effets produits par ces dispositifs de médiatisation au sein de l'espace cultuel (caméras, enceintes, projecteurs, écrans géants, etc.) ne doivent pas être négligés. Ceux-ci participent au déroulement de l'action cérémonielle, écrivent l'image publique du prédicateur, intensifient la relation charismatique et dessinent, dans certaines Églises, les lignes d'une architecture audiovisuelle monumentale qui confère au sermon toute sa dimension de culte-spectacle (fig. 5). Pour toutes ces raisons - dispositifs de médiatisation intégrés à l'action cérémonielle, épidémiologie des représentations, apprentissage par corps, activation et transmission de l'agir divin -, il convient d'entendre par formation télévisuelle le processus continu de métamorphose médiatique de l'expérience pentecôtiste ${ }^{15}$. La médiation télévisuelle est ainsi devenue, par sa technicité même, l'un des principes organisateurs des pentecôtismes contemporains ${ }^{\mathbf{1 6}}$, alors que le rapport des pentecôtistes aux images reste placé sous le signe d'une posture protestante réputée iconophobe.
13. Voir Larbi $2001: 308$ pour la diffusion de l'émission de Roberts au Ghana. Et Lyons et Lyons 1991 pour l'un des premiers articles consacré aux usages pentecôtistes de la télévision au Nigéria et à la figure de Benson Idahosa.

14. Marleen de Witte mentionne également le cas de programmes de variété au Ghana, type "Nouvelle star", où de jeunes apprentis prédicateurs s'affrontent dans une compétition devant un jury composé de prédicateurs confirmés (2012).

15. L'étude des pentecôtismes a significativement contribué au renouvellement des approches sur les relations entre religion et média, depuis le début des années 2000, sous l'angle de la religion comme média. Voir notamment: De Vries et Weber (dir.) 2001 ; Stolow 2005 ;

Engelke 2010 ;

Derrida 2016.

De nombreux chercheurs ont abondé dans ce sens à partir de terrains pentecôtistes africains, particulièrement nigérians et ghanéens: voir Asamoah-Gyadu 2005 ; De Witte 2003 et 2012 ; Meyer et Moors (dir.) 2006 Meyer 2009, 2010 et 2014 Pype 2012 ; Pype et al. 2013 ; Ukah 2003. Pour ce qui est des processus de formation esthétique (aesthetic formations), je renvoie particulièrement à la lecture de Birgit Meyer 2009.

16. Pour une tentative de définition de ces églises comme "media church", voir Mottier 2014b. 


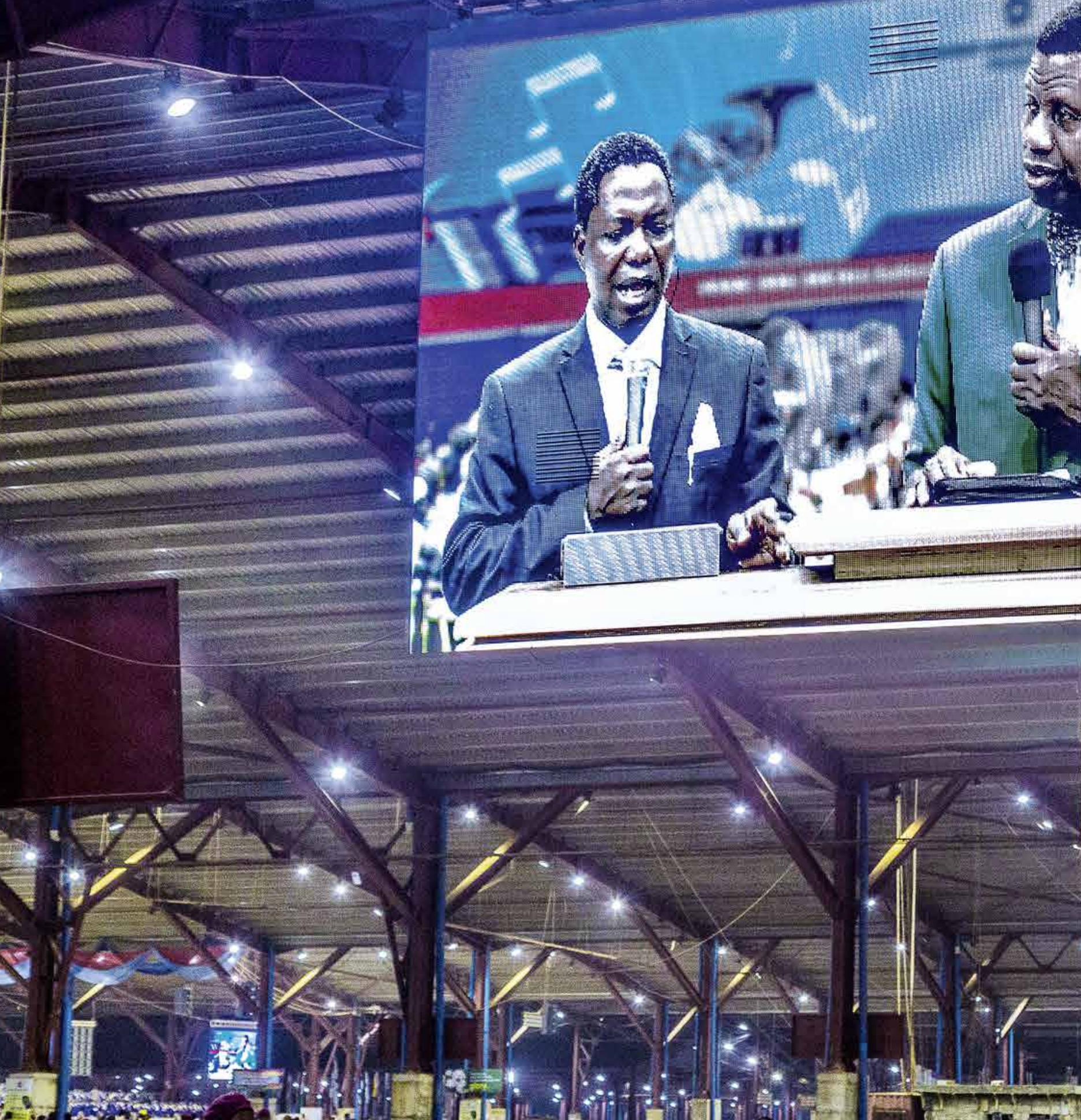

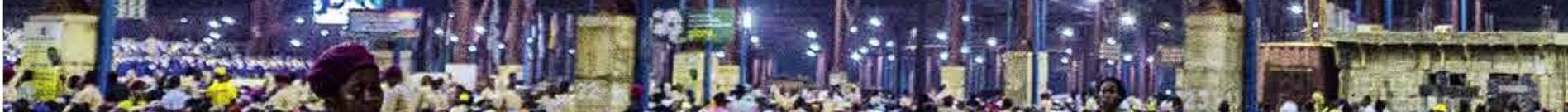

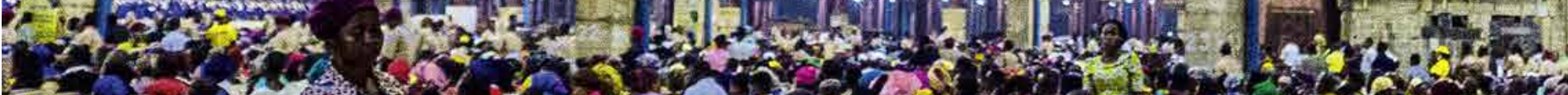

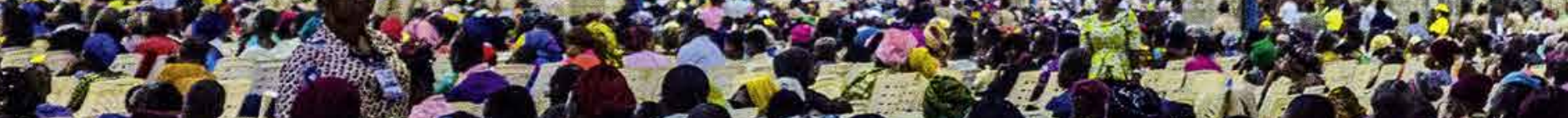
5

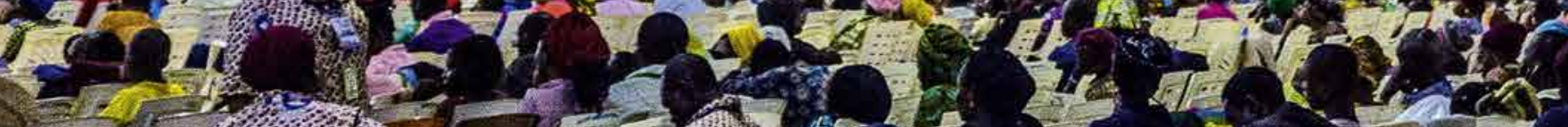

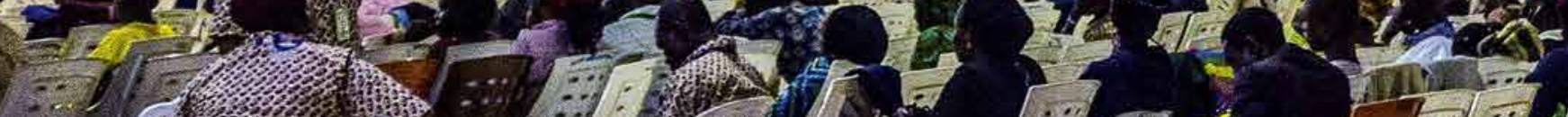

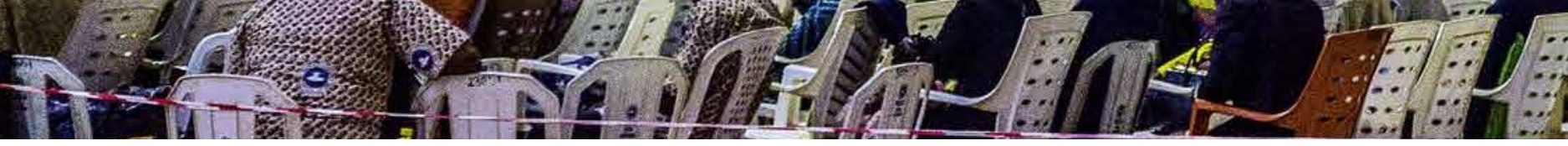




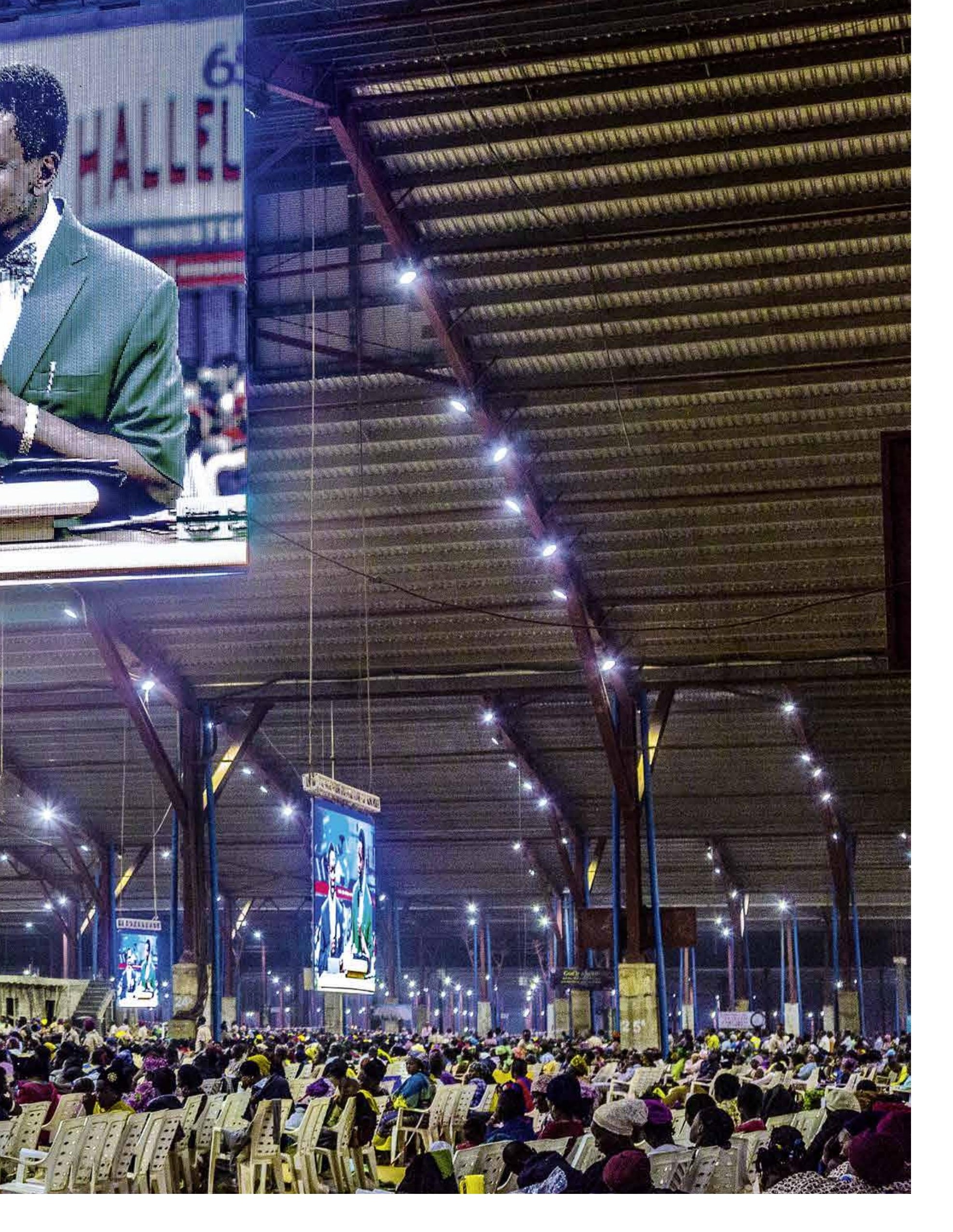


17. Pour plus de détails sur ces figures de prédicateurs, voir Mottier 2014a.

18. Inspirée par les travaux de Jacques Rancière sur la "distribution du sensible», Birgit Meyer a forgé cette notion en lien avec une réflexion qu'elle mène de longue date sur les formes sensationnelles de l'expérience pentecôtiste (2010). double page précédente

fig. 5

Andrew Esiebo, série

God is Alive, Nigéria, 2006

(C) Andrew Esiebo.

\section{ci-contre}

fig. 6

Eugène Irabé

Kacou Severin, série

photographique, 2001

(C) Eugène Irabé.

\section{Corps et images}

Les usages télévisuels des pentecôtistes cohabitent sans contradiction apparente avec cette posture iconophobe pour plusieurs raisons: le réalisme et la transitivité supposés du médium garantissent, comme par transparence, un accès direct à la parole de Dieu animant le corps des prédicateurs; l'effusion du Saint-Esprit peut se réaliser dans le corps des fidèles, à leur domicile, par la vertu des téléprédicateurs; enfin, la disqualification de toute autre tradition iconographique (images peintes, sculptées, etc.) est constante et parfois virulente, comme en témoigne l'épisode bien connu du «coup de pied à la Vierge». Aussi, c'est le corps des prédicateurs, dont les images fixes ou animées prolifèrent sur différents supports (vidéos de sermons, affiches, couvertures de livres, etc.), qui reste à interroger. Le moment est donc venu d'introduire quelques photographies d'une figure majeure des pentecôtismes africains francophones, Kacou Séverin.

Kacou Séverin s'est inscrit à sa manière dans l'héritage institutionnel d'Aimée Semple McPherson en devenant, en 1997, le président de la mission ivoirienne de l'International Church of the Foursquare Gospel que la prédicatrice américaine avait fondée à Los Angeles en 1923. Décédé en avril 2001 dans un accident de voiture à Abidjan à l'âge de 36 ans, alors qu'il bénéficiait d'une renommée internationale grandissante, Kacou Séverin a été le modèle de toute une génération de prédicateurs africains dont j'ai suivi, pour certains, la trajectoire en région parisienne ${ }^{\mathbf{1 7}}$. Dans cette série d'une trentaine de clichés réalisés à Paris, en studio, une semaine avant son décès, il met en scène une certaine conception de sa personnalité charismatique sous l'œil complice du photographe Eugène Irabe (fig. 6). Chaque geste est soigné, réfléchi, accentué: il tient une Bible ouverte entre ses mains, s'agenouille, se relève, serre le poing, pointe du doigt et effectue plusieurs changements de costume. Son corps est le principal cadre d'intelligibilité de sa performance de prédicateur et les rares objets manipulés - la Bible, sa tenue vestimentaire - participent à sa qualification comme système d'actions et de relations. Kacou Séverin s'attache à rendre aussi lisible que possible la profondeur de son animation corporelle, qu'il chorégraphie dans une série de gestes fondamentaux en décomposant les traits communicationnels pertinents de sa vocation. Pourtant, une pose essentielle manque à cette «esthétique de la persuasion ${ }^{\mathbf{1 8}}$ ».

Parmi toutes celles qui m'ont été confiées, aucune photographie ne donne à voir cette «formule d'émotion » qui consiste à offrir la paume de sa main au regard des spectateurs. Deux principaux arguments peuvent être avancés pour justifier cette absence. Le premier consiste à rappeler qu'il s'agit d'une série d'images fixes, dont le principal enjeu était de figurer son charisme et d'illustrer les affiches de ses campagnes d'évangélisation ou les jacquettes de ses vidéos de sermon. S'adaptant aux caractéristiques du médium, Kacou Séverin a ainsi privilégié une grammaire corporelle attachée de longue date aux représentations photographiques des prédicateurs revivalistes: le doigt pointé vers l'objectif du photographe renvoie, par exemple, à quelques célèbres figures du début $d u x x^{e}$ siècle qui ont, elles aussi, pris la pose en studio (fig. 7). Si ce doigt perfore l'image - c'est d'ailleurs pourquoi ce thème a été repris sur de nombreuses affiches de propagande politique pour inciter à l'engagement ou à la conscription (Ginzburg 2013) il n'aimante pas les sujets regardants dans une forme d'étreinte corporelle 


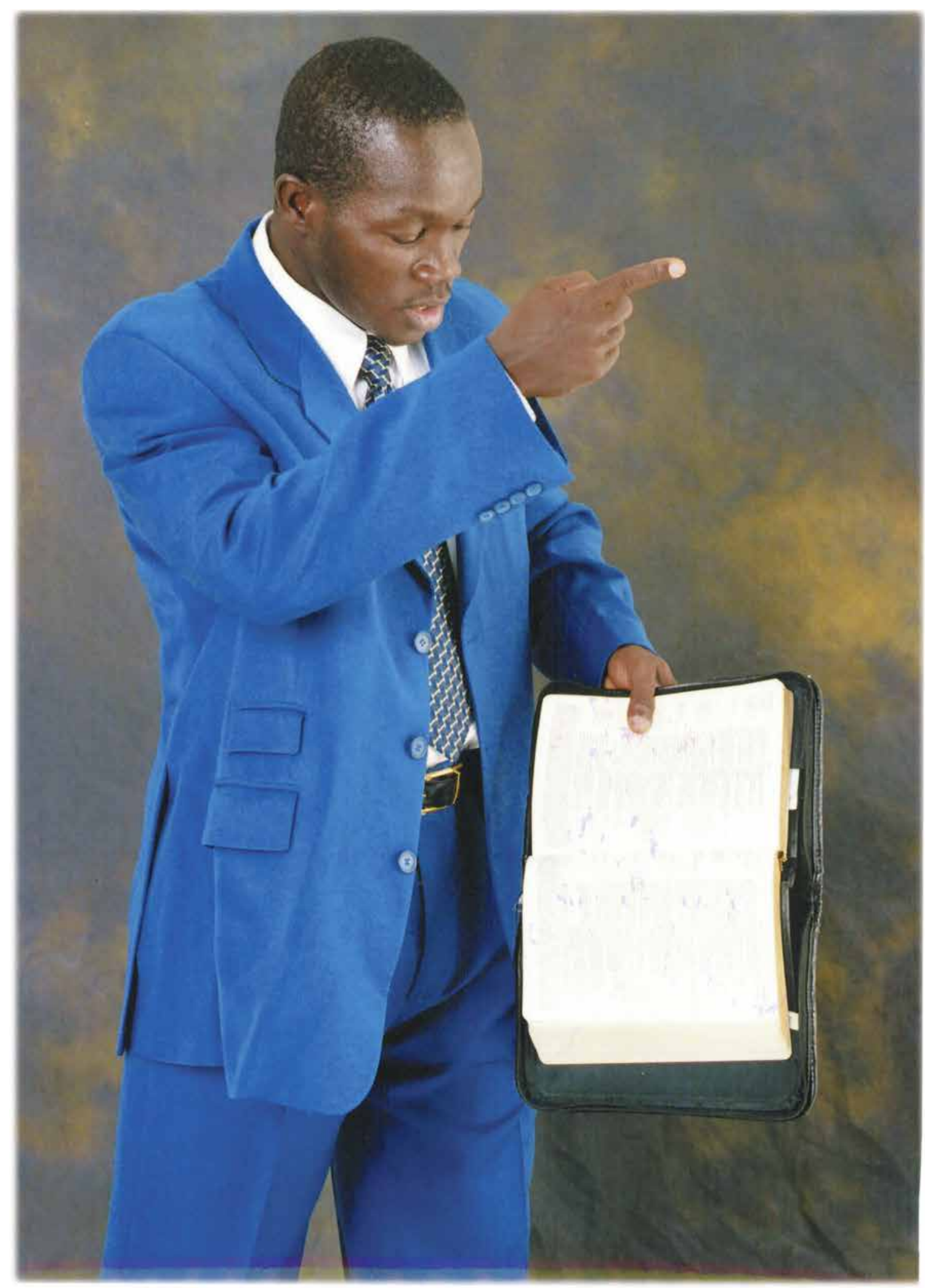




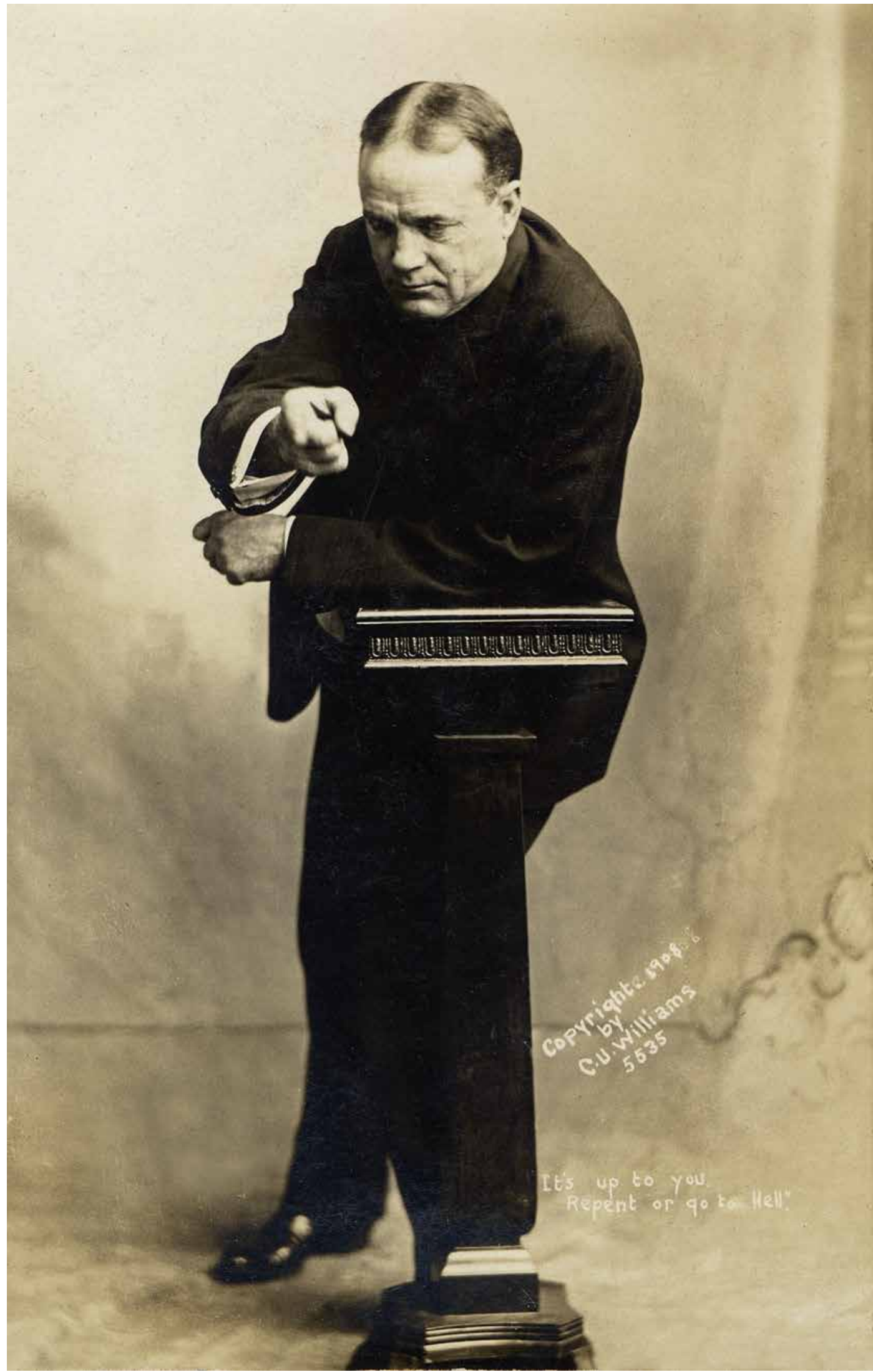


que seule l'animation télévisuelle paraît en mesure d'instaurer techniquement. L'animation manque donc à la photographie pour justifier l'ouverture de la main. Deuxièmement, ce qui est fondamental avec la médiation télévisuelle est qu'elle inscrit, à la différence de la photographie, le Verbe et la Parole dans l'image. Généralement formulée au terme du sermon, l'invitation à poser la main sur l'écran est systématiquement accompagnée par une prière d'autorité. C'est donc par la communication établie oralement avec Dieu grâce à la voix du prédicateur que l'Esprit saint est transmis miraculeusement de la main à la main et qu'il agit à travers ce point de contact pour atteindre les fidèles. Un tel corps-à-corps ne peut être performant sans la Parole animée qui en garantit l'efficacité, sans ce couplage télévisuel du visible et de l'audible et leur recouvrement par le tactile.

Aussi souvent que j'ai observé des situations de visionnement de vidéos de sermons au domicile des fidèles, il est apparu que c'est la voix des prédicateurs qui attire leur regard et appelle leur implication corporelle dans l'image. L'attention des fidèles à leur domicile est généralement flottante - ils ne sont pas nécessairement assis ou positionnés face à leur écran mais vont et viennent selon les circonstances de leur vie domestique. Stimulés par l'ambiance sonore qui prime dans la transmission du message (Rowlands 2007), les fidèles interagissent verbalement - «alléluia, amen, prêche » - sans que leurs regards soient orientés vers l'écran. L'écoute est le principal mode d'engagement dans les sermons télévisés. Et ce n'est qu'au terme d'une prédication convaincante qu'ils sont aimantés par l'image du prédicateur, à l'instar des fidèles présents physiquement au sein de l'espace cultuel qui s'approchent de lui pour recevoir sa bénédiction par imposition des mains. L'expérience du toucher télévisuel épouse donc le rythme du culte filmé et inscrit les fidèles, à domicile, dans un cérémoniel qu'ils connaissent parfaitement.

Conditionnée par la durée de la prédication et soutenue par l'incantation du prédicateur, la main posée sur l'écran - parfois simplement orientée vers l'écran ou posée sur soi - scelle un acte majeur de l'expérience pentecôtiste: la communion par l'Esprit saint qui, donnée à tous en partage, est au fondement de cette communauté d'expérience. La possibilité technique de cette communion distingue radicalement les usages pentecôtistes de la médiation télévisuelle d'autres traditions religieuses, les catholiques ne pouvant par exemple concevoir une telle distribution télévisuelle de l'Eucharistie. L'imaginaire pentecôtiste de l'agir divin est ainsi réalisé par cette technique du corps-à-corps télévisuel qui participe à l'instauration d'une vision organique et réticulaire de l'Église, résolument anti-institutionnelle, fondée sur l'intercorporéité et l'intersubjectivité de ceux qui partagent ici et maintenant l'expérience miraculeuse de la Pentecôte.

\section{Empreindre}

Une compréhension de ce geste doit cependant aller au-delà des caractéristiques du médium pour plusieurs raisons. La première est qu'il serait abusif de considérer les usages religieux des "nouveaux médias» comme résolument «nouveaux » sans prendre le soin de les inscrire dans une histoire circonstanciée des formes de l'expérience religieuse. Poser la main sur l'écran est la formulation télévisuelle d'un geste qui s'appuie sur toute une

\section{ci-contre}

fig. 7

C. U. Williams,

Bloomington, III

Billy Sunday en studio,

1908. Carte postale

intitulée: "It's up to you.

Repent or go to hell".

Grace College \& Theological

Seminary, coll. Winona

Lake Postcards. 


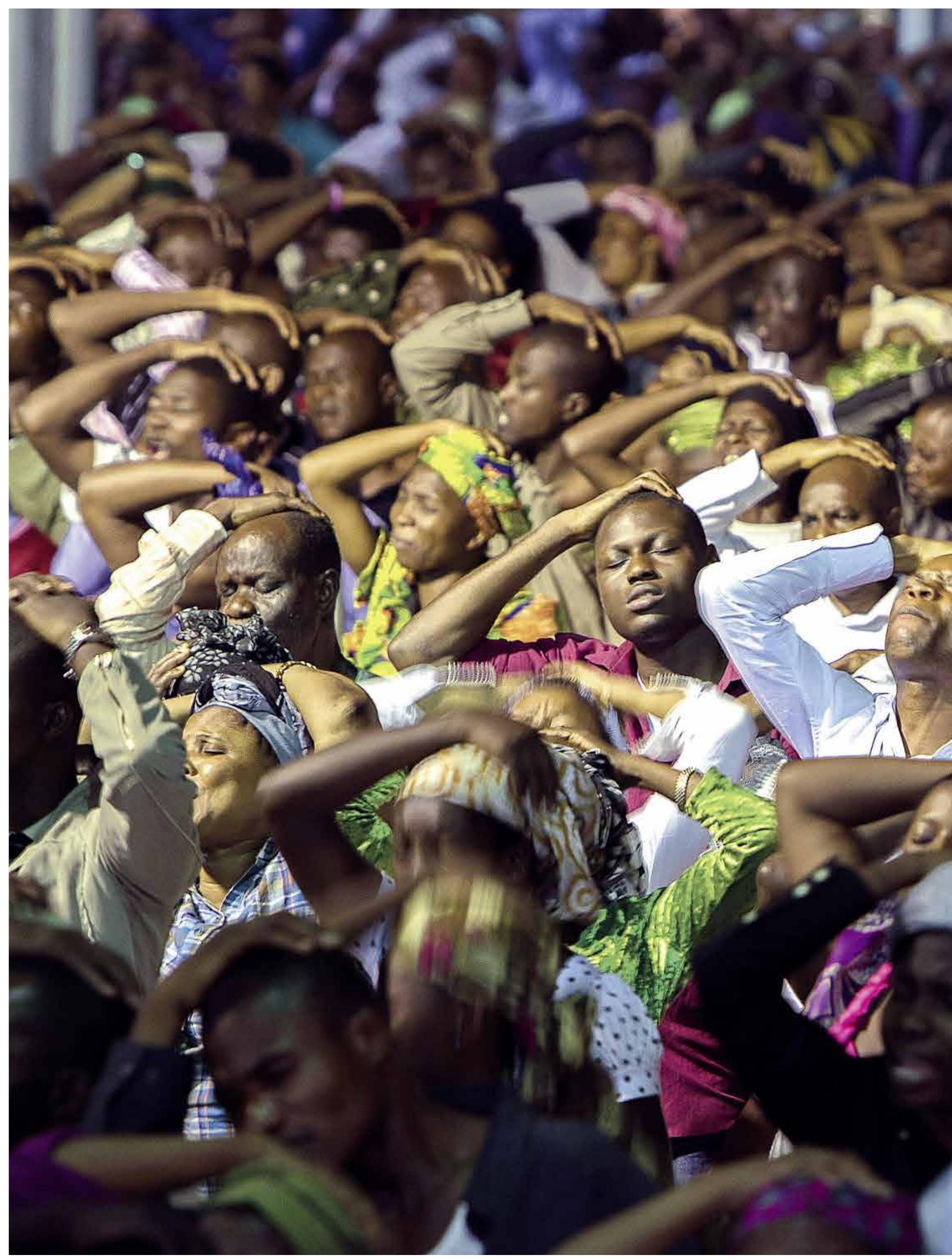



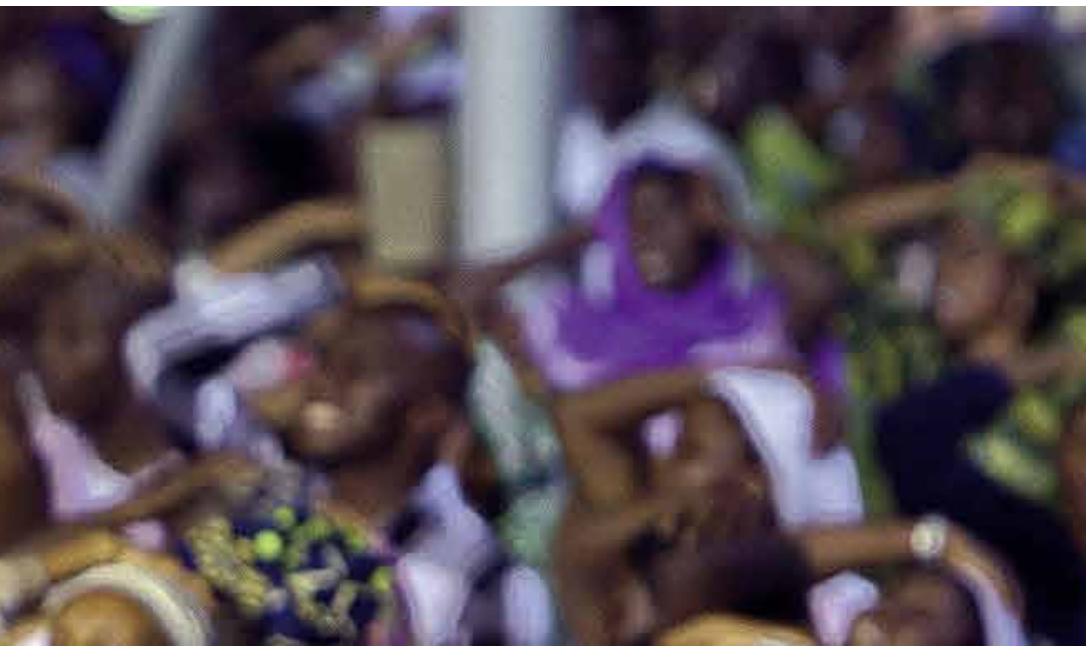

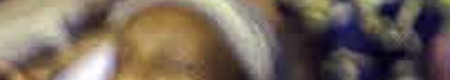

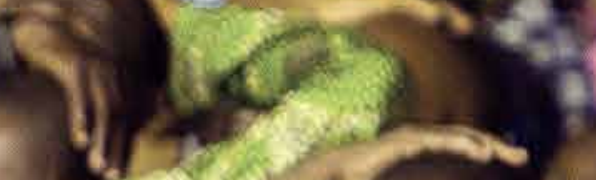

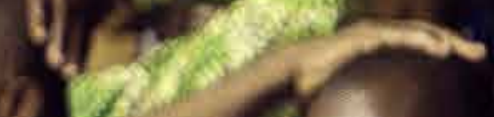
$(1)$ xily S yntro?

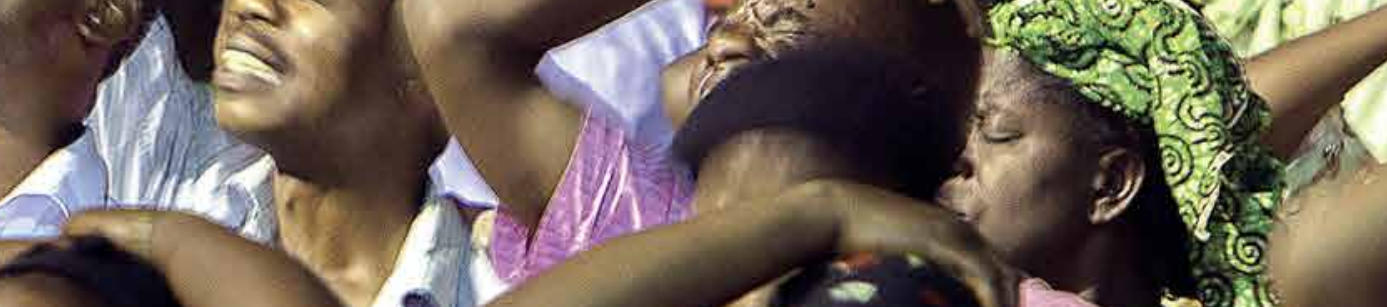

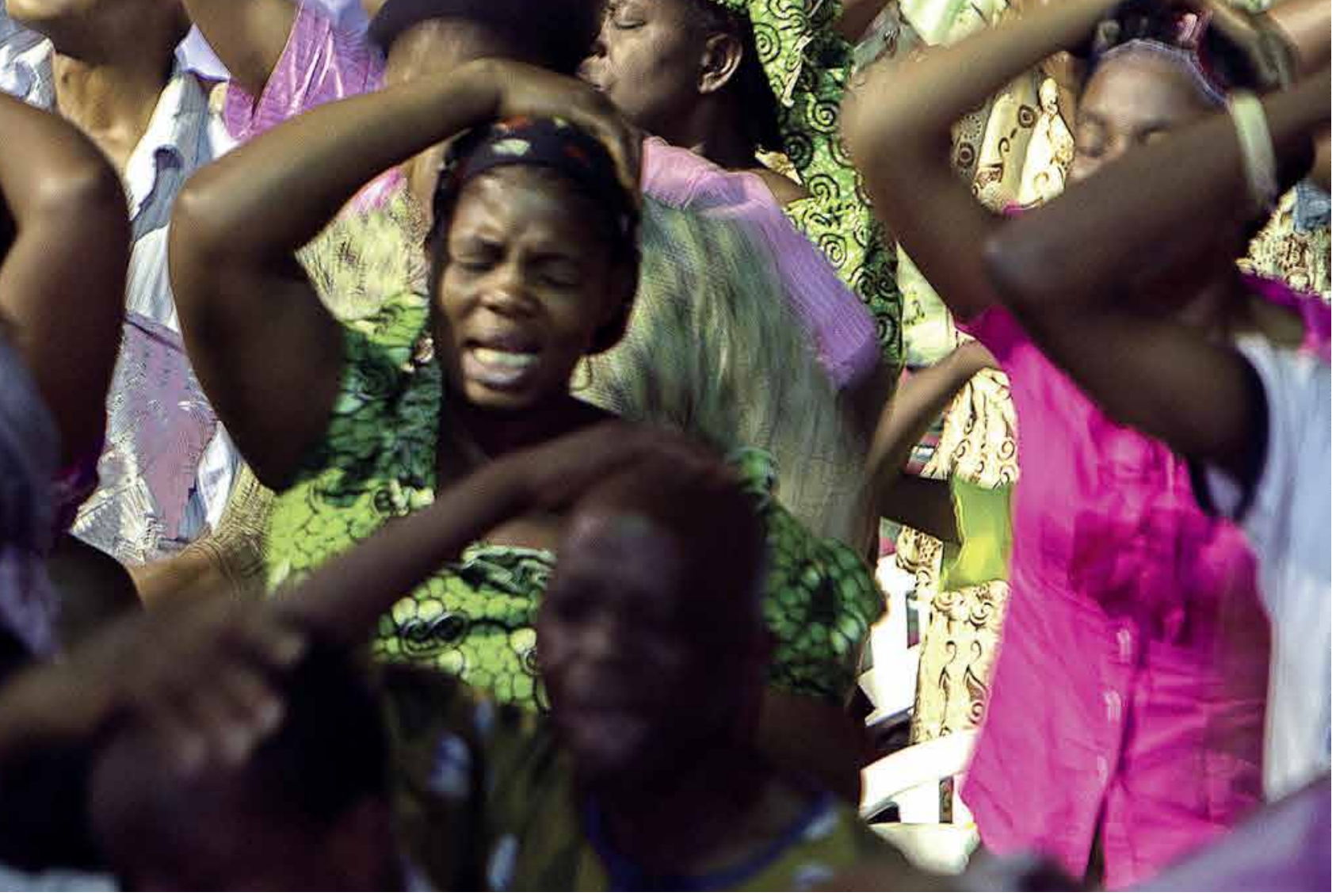


19. Plusieurs scandales sexuels liés à l'imposition des mains et aux techniques de manipulation des corps ont émaillé l'histoire des pentecôtismes. Plus généralement, sur l'érotisme du toucher, voir Benthien 2002

20. De portée générale, cette observation est présente chez Mauss au sujet des techniques du corps mystique comme "moyens biologiques d'entrer en" communication avec le Dieu" " (1999 [1936]

386). Voir également Adell 2015.

21. L'analyse de cet accord est au fondement de l'anthropologie des images selon Hans Belting (2004).

\section{double page précédente}

fig. 8

Andrew Esiebo, série

God is Alive, Nigéria, 2006

(C) Andrew Esiebo. série de pratiques préalables. L'importance des sermons radiodiffusés, par exemple, ne doit pas être négligée: dès les années 1920, ceux-ci ont imposé l'idée que le Saint-Esprit pouvait agir au-delà du cercle des croyants réunis en un même lieu et être activé par les ondes radiophoniques - la main des auditeurs étant parfois orientée vers le poste de radiodiffusion à la demande du prédicateur. Plus généralement, l'imposition directe de la main du prédicateur sur différentes parties du corps des fidèles (le front en particulier) est un geste caractéristique des faith healers - certains prédicateurs privilégiant cependant la diffusion du Saint-Esprit dans «l'atmosphère» pour éviter de manipuler directement les fidèles et dissiper, ainsi, toute forme d'ambiguïté liée à l'érotisme du toucher ${ }^{19}$. Enfin, les mains des fidèles sont particulièrement actives durant les cultes pour ouvrir les corps à une communication avec l'Esprit saint et orienter son action (fig. 8). Leurs positions se déploient selon d'infinies nuances: qu'il s'agisse d'une paume ouverte en direction du prédicateur pour recevoir individuellement la Parole de Dieu qui sort de sa bouche, d'une main orientée vers le ciel, tournée vers soi ou posée sur une partie du corps souffrant durant une prière, sinon d'une main qui ventile le visage pour rapprocher de soi la manifestation du Saint-Esprit diffus dans l'atmosphère.

Poser la main sur l'écran n'est donc pas un acte isolé mais une manifestation de la «simplexité » des modes d'expérimentation pentecôtistes, autrement dit «un résumé immédiatement compréhensible d'une réalité complexe» (Berthoz 2009: 129). Et ce qui est frappant dans l'activité de la main - la main constituant l'organe privilégié de la communication avec l'esprit 20 _ est que le toucher éteint le plus souvent le regard. Les yeux se ferment lorsque les mains s'ouvrent et se joignent dans la prière pour faciliter cette prise de corps tandis que le volume de la voix augmente pour accompagner cette déflagration intime. Cette expérience de la manifestation de l'Esprit fait corps est généralement liée à un tel basculement des registres sensoriels. Et c'est probablement ce décrochage dans l'ordonnancement des stimulations perceptuelles qui, inscrit dans la temporalité de l'action rituelle, fait de la main posée sur l'écran un symptôme de l'expérience pentecôtiste multipliant les possibilités d'être affecté. II ne s'agit pas d'être touché par le regard ou la voix du prédicateur, mais d'être transformé par la vision incorporée de l'Esprit saint.

Deuxième raison en faveur d'une compréhension renouvelée de ce geste allant au-delà des caractéristiques du médium - et qui vaudra pour conclusion provisoire - une ethnographie de cette «formule d'émotion » doit nécessairement s'inscrire dans une anthropologie historique des images, qu'elle soit attachée au temps long du christianisme médiéval ou à une conception de l'histoire d'inspiration warburgienne. L'exercice de comparaison est délicat, certes, et inquiétant, mais l'attention portée aux modalités de l'engagement du corps dans l'image peut nous y aider. «Tout change pour que rien ne change», écrivait Giuseppe Tomasi di Lampedusa (1958). Telle pourrait être la modeste conclusion de cet article. Car malgré l'attitude ambivalente des pentecôtistes face aux images matérielles - et les images télévisuelles sont des images matérielles sauf à nier la matérialité en trois dimensions des différents écrans dans lesquels se meuvent les corps animés des prédicateurs -, le principe de la culture de l'imago demeure dans un accord corps-image-médium ${ }^{\mathbf{2 1}}$ métamorphosé et reformulé selon les modalités de l'expérience pentecôtiste. Les trois «domaines de l'imago 
médiévale" sont: "Celui des images matérielles (imagines) ; celui de l'imaginaire (imaginatio) fait d'images mentales, oniriques et poétiques ; et enfin celui de l'anthro-pologie et de la théologie chrétiennes, fondées sur une conception de l'homme créé ad imagem Dei et promis au salut par l'Incarnation du Christ imago Patris. (Schmitt 2002: 54) Quel motif de l'Incarnation plus concret que cette prise de corps télévisuelle? Le contact est établi charnellement avec l'Esprit saint par application de la main sur la surface d'une image techniquement vivante, mettant en jeu le corps d'un activateur de charisme - une «icône charismatique», écrivent certains chercheurs (Meyer 2009: 15) selon un couplage image-corps qui n'est pas sans faire penser aux statuesreliquaires et aux matières organiques de saints qui étaient parfois «scellées dans la tête ou dans le ventre de certaines images" (Schmitt 2002 : 26), ou encore, pour prendre un exemple plus récent, aux liens charnels que certaines mystiques libanaises contemporaines entretiennent avec des images de la Vierge (Aubin-Boltanski 2016).

Enfin, comment résister à une histoire anachronique des formes? Comment ne pas suggérer un dernier et vertigineux déplacement, hors du champ chrétien, en rappelant que les plus anciennes images projetées que nous connaissons sont des mains inscrites sur les parois des grottes. Ces «mains premières", selon l'expression d'André Breton (1962), parfois négatives, sont soufflées, c'est-à-dire animées techniquement. Une partie du problème est peut-être de n'y voir qu'une empreinte alors que c'est le corps lui-même qui est empreint, "pris" - initié ou converti -, dans un système d'actions. L'image est un geste. Et c'est ce geste qu'il faut saisir pour atteindre l'image.

Pour conclure, cette fois définitivement, un article qui pourrait appeler d'autres développements, je dirais que la télévision, pas plus que la culture médiévale de l'imago, ne se réduit à un simple objet matériel. La matérialité des écrans (plats ou bombés, en noir et blanc ou en couleur), leurs caractéristiques techniques (des tubes cathodiques aux écrans miniatures des smartphones), leur monumentalité ou leur transportabilité (d'un élément de mobilier occupant une place centrale dans l'espace domestique aux objets portatifs en toutes circonstances) sont évidemment à interroger pour accompagner au plus près les vicissitudes de cette mutation formelle du touché-touchant télévisuel qui inscrit la tradition pentecôtiste, en dépit d'une posture réputée iconophobe, dans une pensée visuelle de l'Incarnation. La télévision est "vision à distance"; à moins que ce ne soit la vision elle-même qui est toujours, nécessairement, une télé-vision, comme l'écrit Merleau-Ponty dans une note rédigée en mars 1961, trois mois avant l'étreinte manquée de Richard main contre main avec Roberts par laquelle j'ai ouvert cet article: «la vision est télé-vision, transcendance, cristallisation de l'impossible» (Merleau-Ponty 2015 [1964]: 321). Et c'est probablement cet écart, cette déchirure - si près, si loin - qui justifie l'expérience du toucher télévisuel, dès lors que la relation au visible est investie des puissances de l'imagination religieuse. 
Bibliographie

\section{Adell, Nicolas}

2015 «Introduction: La part de la main. Des rapports entre la main et l'esprit en anthropologie", ethnographiques.org 31 [en ligne], disponible sur: http://www. ethnographiques.org/2015/Adell

\section{Aubin-Boltanski, Emma}

2016 "La très petite image de Notre-Dame de Soufanieh", Archives de sciences sociales des religions 174: 101-123.

\section{Asamoah-Gyadu, Kwabena}

2005 "Anointing Through the Screen: Neo-Pentecostalism and Televised Christianity in Ghana ", Studies in World Christianity 11 (1): 9-28.

\section{Belting, Hans}

2004 Pour une anthropologie des images, trad. de l'allemand par Jean Torrent. Paris, Gallimard.

\section{Benthien, Claudia}

2002 "Teletactility: the Skin in New Media », in Claudia Benthien, Skin: On the Cultural Border Between Self and World. Chichester, Colombia University Press : 221-234.

\section{Berthoz, Alain}

2009 La Simplexité. Paris, Odile Jacob.

\section{Breton, André}

1962 "Main première", in Karel Kupka, Un art à l'état brut: peintures et sculptures des aborigènes d'Australie. Lausanne, Clairefontaine.

\section{Cassaniti, Julia et} Lurhmann, Tanya

2014 "The Cultural Kindling of Spiritual Experiences », Current Anthropology 55 (10) : 333-343.

\section{Chidester, David}

$\mathbf{2 0 0 0}$ "Haptics of the Heart: the Sense of Touch in American Religion and Culture ", Culture and Religion 1 (1) : 6I-84

2005 «The American Touch. Tactile Imagery in American Religion and Politics ", in Constance Classen (dir.), The Book of Touch. Oxford/ New York, Berg: 49-65

La main sur l'écran. Pentecôtisme et expérience du "toucher" télévisuel.

Par Damien Mottier

\section{Csordas, Thomas}

1997 The Sacred Self: a Cultural Phenomenology of Charismatic Healing. Berkeley/Los Angeles/ Londres, University of California Press.

2002 Body/Meaning/Healing. New York, Palgrave Macmillan.

2009 Transnational transcendence Essays on Religion and Globalization. Berkeley/Los Angeles/Londres, University of California Press.

\section{De Abreu, Maria Jose}

2009 «Breath, Technology, Making of Community ", in Birgit Meyer (dir.) Aesthetic formations: Media, Religion and the Senses. New York

Palgrave Macmillan: 161-182.

\section{Derrida, Jacques}

2000 Le Toucher: Jean-Luc Nancy. Paris, Galilée.

2016 Surtout, pas de journalistes! Paris, Galilée.

\section{De Witte, Marleen}

2003 "Altar Media's Living Word: Televised Charismatic Christianity in Ghana ", Journal of religion in Africa 33 (2) : 172-202.

2011a "Touched by the Spirit : Converting the Senses in a Ghanaian Charismatic Church ", Ethnos 76 (4) 489-509.

2011b "Touch», Material Religion 7 (1) : 148-155.

2012 «Television and the Gospe of Entertainment in Ghana ", Exchange 41 (2) : 144-164

2013 «The Electric Touch Machine Miracle Scam: Body, Technology, and the (Dis)authentication of the Pentecostal Supernatural ", in Jeremy Stolow (dir.), Deus in Machina: Religion, Technology, and the Things in Between. New York, Fordham University Press : 61-82.

De Vries, Hent et Weber, Samuel (dir.)

2001 Religion and Media. Stanford Stanford University Press.

\section{Didi-Huberman, Georges}

2007 L'Image ouverte: motifs de l'incarnation dans les arts visuels. Paris, Gallimard.
2008 La Ressemblance par contact: archéologie, anachronisme et modernité de l'empreinte. Paris, Les Éditions de Minuit.

\section{Engelke, Matthew}

2010 «Religion and the Media Turn : a Review Essay ", American ethnologist 37 (2) : 371-379.

\section{Ginzburg, Carlo}

2013 Peur révérence terreur: quatre essais d'iconographie politique, trad. de l'anglais et de l'italien par Martin Rueff.

Dijon, Les presses du réel.

\section{Harrel, David Edwin}

1985 Oral Roberts : an American Life. Bloomington, Indiana University Press.

\section{Larbi, Emmanuel Kingsley}

2001 Pentecostalism : the Eddies of Ghanaian Christianity. Accra,

Center for Pentecostal and

Charismatic Studies.

\section{Leventopoulos, Mélisande}

2011 "Une Église moderne en images: la cause cinématographique du père Raymond Pichard (1947-1954) », 1895: 70-89.

\section{Lurhmann, Tanya}

2012 When God Talks Back

Understanding the American

Evangelical Relationship with God.

New York, Alfred E. Knopf.

\section{Lyons, Andrew et Lyons, Harriet}

1991 "Religion and the Mass Media in Nigeria ", in Jacaob Olupona (dir.) Religion and Society in Nigeria. Ibadan, Spectrum: 97-128.

\section{Mauss, Marcel}

1999 [1936] "Les techniques du corps ", in Marcel Mauss, Sociologie et anthropologie. Paris, PUF : 363-386.

\section{Merleau-Ponty, Maurice}

2015 [1964] Le Visible et l'invisible. Paris, Gallimard.

\section{Meyer, Birgit}

2009 Aesthetic formation: Media Religion and the Senses. New York Palgrave Macmillan.
2010 «Aesthetics of Persuasion: Global Christianity and

Pentecostalism's Sensationa

Forms ", South Atlantic

Quarterly 109 (4) : 741-763.

2014 «Mediation and the Genesis of Presence", Religion and Society Advances in Research 5: 205-254.

Meyer, Birgit et Moors, Annelies (dir.)

2006 Religion, Media, and the Public Sphere. Bloomington, Indiana University Press.

\section{Mottier, Damien}

2014a Une ethnographie des pentecôtismes africains en France le temps des prophètes.

Louvain-la-Neuve,

Academia-L'Harmattan.

2014b "Media-church:

ethnographie des dispositifs de médiatisation en milieu pentecôtiste charismatique ", MEI 38: 141-151.

\section{Pype, Katrien}

2012 The Making of Pentecostal Melodrama. Religion, Media and Gender in Kinshasa. New York/ Oxford, Berghahn Books.

Pype, Katrien, Mélice, Anne et Van Wolputte, Steven

2013 "Introduction: Visions du Paradis ", Social Compass 61 (1) : 3-10.

\section{Rowlands, Michael}

2007 "The Sound of Witchcraft : Noise as Mediation in Religious Transmission ", in David Berliner et Ramon Sarró (dir.), Learning Religion: Anthropological Approaches. New York/Oxford, Berghahn Books: 191-208.

\section{Schmitt, Jean-Claude}

2002 Le Corps des images: essais sur la culture visuelle au Moyen Âge. Paris, Gallimard.

\section{Stolow, Jeremy}

2005 "Religion and/as Media ", Theory, Culture \& Society 22 (4) 119-145. 


\section{Sutton, Matthew Avery}

2007 Aimee Semple McPherson and the Resurrection of Christian America. Cambridge, Harvard University Press.

\section{Tomasi di Lampedusa, Giuseppe}

1958 Le Guépard. Paris, Seuil.

\section{Ukah, Asonzeh}

2003 "Advertising God: Nigerian Christian Video-Films and the Power of Consumer Culture ", Journal of Religion in Africa 33 (2) : 203-231.

\section{Willaime, Jean-Paul}

1999 «Le pentecôtisme: contours et paradoxes d'un protestantisme émotionnel ", Archives de sciences sociales des religions 105 (1) : 5-28.

page 22 et ci-contre Oral Roberts, détail, reportage "Le chapiteau des miracles",

Cinq colonnes à la une, ORTF, 2 juin 1961. DR.

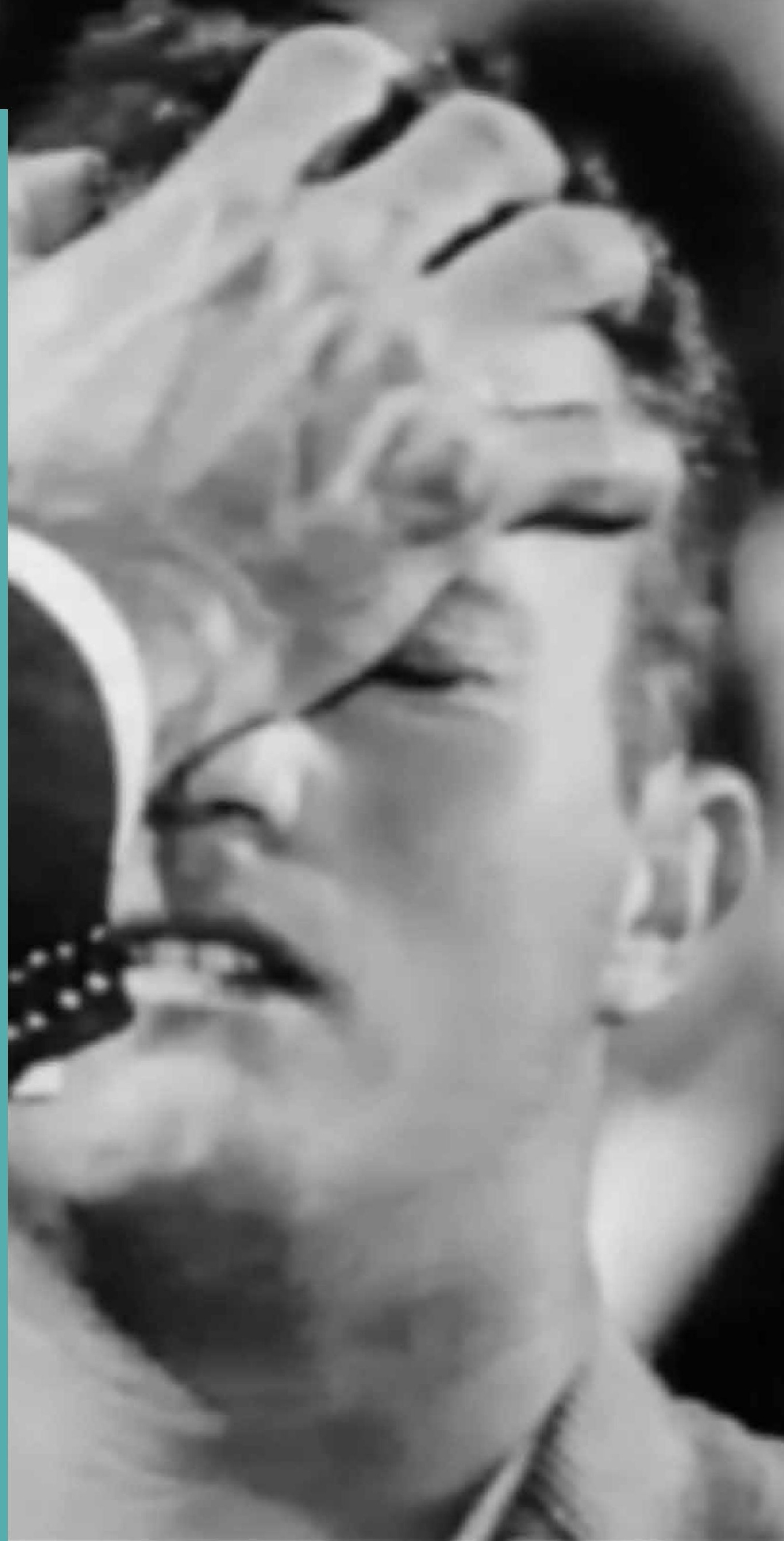

